\title{
Putting Mechanics into Quantum Mechanics
}

\section{Nanoelectromechanical structures are starting to approach the ultimate quantum mechanical limits for detecting and exciting motion at the nanoscale. Nonclassical states of a mechanical resonator are also on the horizon.}

\author{
Keith C. Schwab and Michael L. Roukes
}

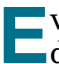
verything moves! In a world dominated by electronic devices and instruments it is easy to forget that all measurements involve motion, whether it be the motion of electrons through a transistor, Cooper pairs or quasiparticles through a superconducting quantum interference device (SQUID), photons through an optical interferometer-or the simple displacement of a mechanical element. Nanoscience today is driving a resurgence of interest in mechanical devices, which have long been used as front ends for sensitive force detectors. Among prominent historical examples are Coulomb's mechanical torsion balance, which allowed him in 1785 to establish the inversesquare force law of electric charges, and Cavendish's mechanical instrument that allowed him in 1798 to measure the gravitational force between two lead spheres.

Today, micro- and nanoelectromechanical systems (MEMS and NEMS) are widely employed in ways similar to those early force detectors, yet with vastly greater force and mass sensitivity-now pushing into the realm of zeptonewtons $\left(10^{-21} \mathrm{~N}\right)$ and zeptograms $\left(10^{-21} \mathrm{~g}\right)$. These ultraminiature sensors also can provide spatial resolution at the atomic scale and vibrate at frequencies in the gigahertz range. ${ }^{1}$ Among the breadth of applications that have become possible are measurements of forces between individual biomolecules, ${ }^{2}$ forces arising from magnetic resonance of single spins, ${ }^{3}$ and perturbations that arise from mass fluctuations involving single atoms and molecules. ${ }^{4}$ The patterning of mechanical structures with nanometerscale features is now commonplace; figure 1 and the cover display examples of current devices.

The technological future for small mechanical devices clearly seems bright, yet some of the most intriguing applications of NEMS remain squarely within the realm of fundamental research. Although the sensors for the applications mentioned above are governed by classical physics, the imprint of quantum phenomena upon them can now be readily seen in the laboratory. For example, the Casimir effect, arising from the zero-point fluctuations of the electromagnetic vacuum, can drive certain small mechanical devices with a force of hundreds of piconewtons and produce discernible motion in the devices. ${ }^{5}$ But it is now possible, and perhaps even more intriguing, to consider the intrinsic quantum fluctuations-those that belong to the mechanical device itself. The continual progress in shrinking devices, and the profound increases in sensitivity

Keith Schwab is a senior physicist at the National Security Agency's Laboratory for Physical Sciences in College Park, Maryland. Michael Roukes is Director of the Kavli Nanoscience Institute and is a professor of physics, applied physics, and bioengineering at the California Institute of Technology in Pasadena. achieved to read out those devices, now bring us to the realm of quantum mechanical systems.

\section{The quantum realm}

What conditions are required to observe the quantum properties of a mechanical structure, and what can we learn when we encounter them? Such questions have received considerable attention from the community pursuing gravitational-wave detection: For more than 25 years, that community has understood that the quantum properties of mechanical detectors will impose ultimate limits on their force sensitivity ${ }^{6,7}$ Through heroic and sustained efforts, the Laser Interferometer Gravitational Wave Observatory (LIGO) with a 10-kg test mass, and cryogenic acoustic detectors with test masses as large as $1000 \mathrm{~kg}$, currently achieve displacement sensitivities only a factor of about 30 from the limits set by the uncertainty principle.

But the quantum-engineering considerations for mechanical detectors are not exclusive to the realm of gravitational-wave physics. In the introduction to their pioneering book on quantum measurement, Vladimir Braginsky and Farid Khalili envisage an era when quantum considerations become central to much of commercial engineering. ${ }^{7}$ Today, we are approaching that timeadvances in the sensitivity of force detection for new types of scanning force microscopy point to an era when mechanical engineers will have to include $\hbar$ among their list of standard engineering constants.

Several laboratories worldwide are pursuing mechanical detection of single nuclear spins. That goal is especially compelling in light of the recent success of Dan Rugar and colleagues at IBM in detecting a single electron spin with a MEMS device (see figure $1 \mathrm{~b}$ and PHYSICS TODAY, October 2004, page 22). ${ }^{3}$ However, nuclear spins generate mechanical forces of about $10^{-21} \mathrm{~N}$, more than 1000 times smaller than the forces from single electron spins. One ultimate application of this technique, structural imaging of individual proteins, will involve millions of bits of data and require measurements to be carried out not on the current time scale of hours, but over microseconds. Detecting such small forces within an appropriate measurement time will necessitate new quantum measurement schemes at high frequencies, a significant challenge. Yet the payoff, originally envisaged by John Sidles, will be proportionately immense: three-dimensional, chemically specific atomic imaging of individual macromolecules. ${ }^{8}$

The direct study of quantum mechanics in micron- and submicron-scale mechanical structures is every bit as attractive as the actual applications of MEMS and NEMS. ${ }^{9}$ With resonant frequencies from kilohertz to gigahertz, low 

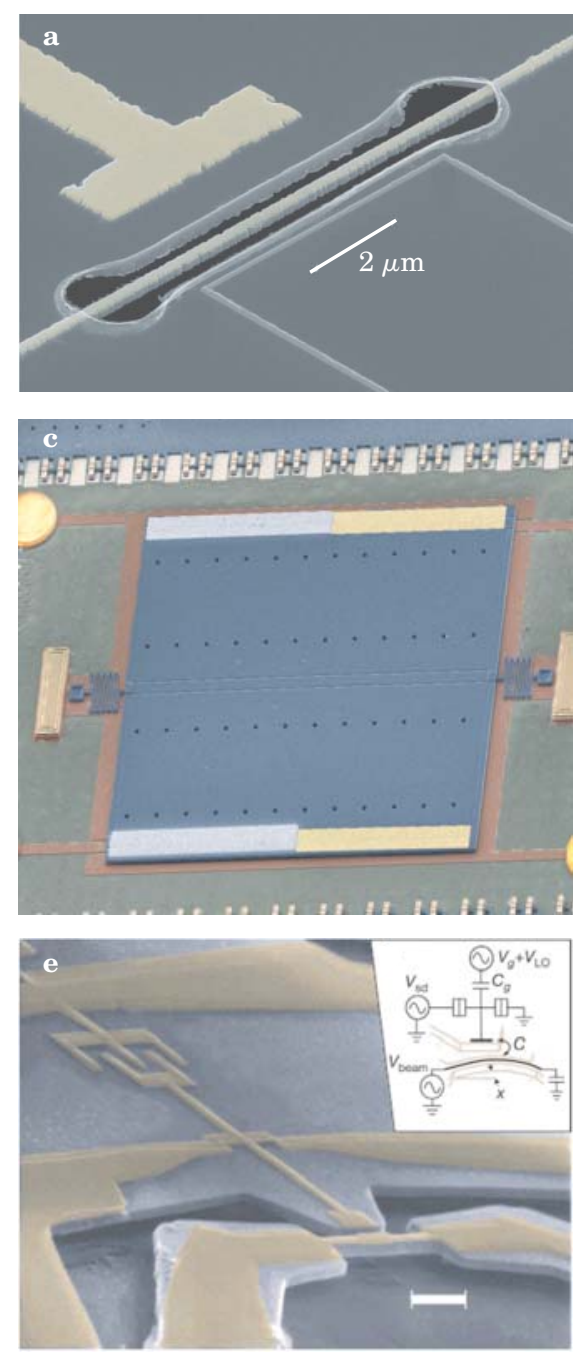
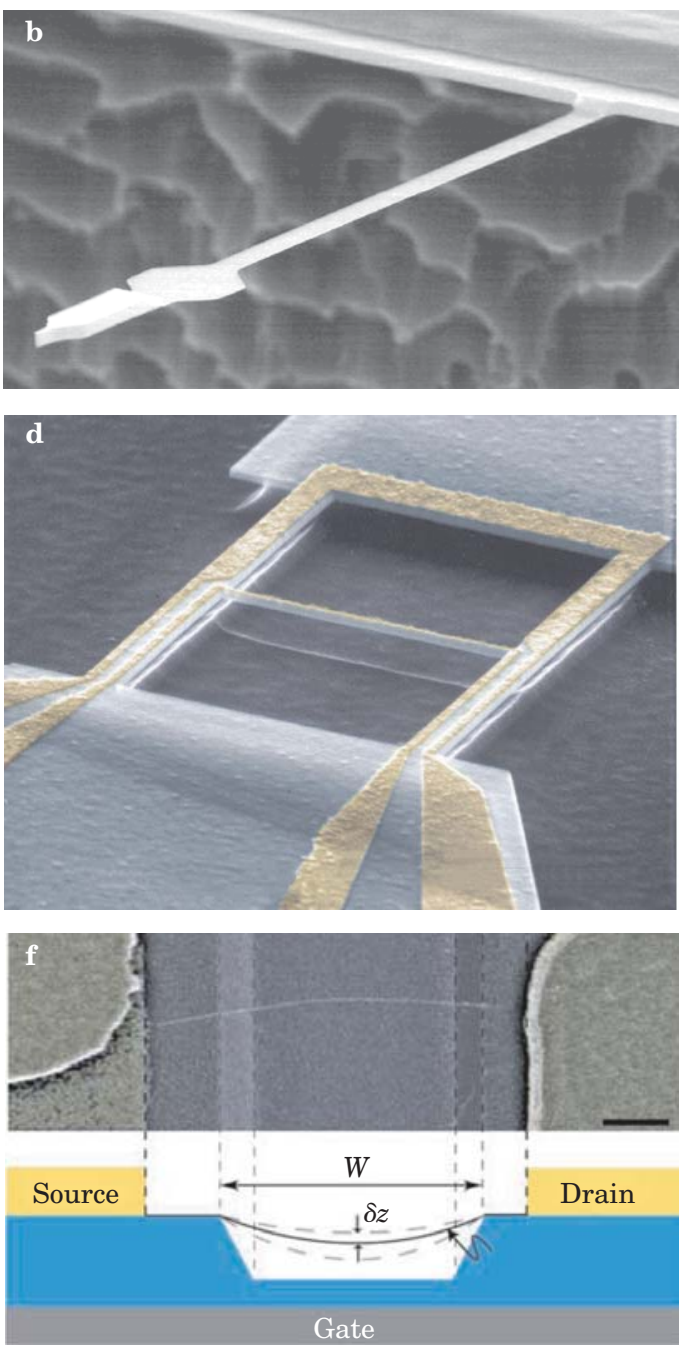

Figure 1. Nanoelectromechanical devices.

(a) A 20-MHz nanomechanical resonator capacitively coupled to a single-electron transistor (Keith Schwab, Laboratory for Physical Sciences). ${ }^{11}$ (b) An ultrasensitive magnetic force detector that has been used to detect a single electron spin (Dan Rugar, IBM). ${ }^{3}$ (c) A torsional resonator used to study Casimir forces and look for possible corrections to Newtonian gravitation at short length scales (Ricardo Decca, Indiana UniversityPurdue University Indianapolis). (d) A parametric radio-frequency mechanical amplifier that provides a thousandfold boost of signal displacements at $17 \mathrm{MHz}$ (Michael Roukes, Caltech). (e) A $116-\mathrm{MHz}$ nanomechanical resonator coupled to a single-electron transistor (Andrew Cleland, University of California, Santa Barbara). ${ }^{10}$

(f) A tunable carbon nanotube resonator operating at $3-300 \mathrm{MHz}$ (Paul McEuen, Cornell University). ${ }^{14}$ dissipation, and small masses $\left(10^{-15}-10^{-17} \mathrm{~kg}\right)$, these devices are well suited to such explorations. Their dimensions not only make them susceptible to local forces, but also make it possible to integrate and tightly couple them to a variety of interesting electronic structures, such as solid-state twolevel systems (quantum bits, or qubits), that exhibit quantum mechanical coherence. In fact, the most-studied systems, nanoresonators coupled to various superconducting qubits, are closely analogous to cavity quantum electrodynamics, although they are realized in a very different parameter space.

\section{Quantized nanomechanical resonators}

The classical and quantum descriptions of a mechanical resonator are very similar to those of the electromagnetic field in a dielectric cavity: The position- and time- dependent mechanical displacement $\mathbf{u}(\mathbf{r}, t)$ is the dynamical variable analogous to the vector potential $\mathbf{A}(\mathbf{r}, t)$. In each case, a wave equation constrained by boundary conditions gives rise to a spectrum of discrete modes. For sufficiently low excitation amplitudes, for which nonlinearities can be ignored, the energy of each mode is quadratic in both the displacement and momentum, and the system can be described as essentially independent simple harmonic oscillators.

Spatially extended mechanical devices, such as those in figure 1 , possess a total of $3 A$ modes of oscillation, where
$A$ is the number of atoms in the structure. Knowing the amplitude and phase of all the mechanical modes is equivalent to having complete knowledge of the position and momentum of every atom in the device. Continuum mechanics, with bulk parameters such as density and Young's modulus, provides an excellent description of the mode structure and the classical dynamics, because the wavelengths $(100 \mathrm{~nm}-10 \mu \mathrm{m})$ of the lowest-lying vibrational modes are long compared to the interatomic spacing.

It is natural to make the distinction between nanomechanical modes and phonons: The former are lowfrequency, long-wavelength modes strongly affected by the boundary conditions of the nanodevice, whereas the latter are vibrational modes with wavelengths much smaller than typical device dimensions. Phonons are relatively unaffected by the geometry of the resonator and, except in devices such as nanotubes that approach atomic dimensions, are essentially identical in nature to phonons in an infinite medium.

It is an assumption that quantum mechanics should even apply for such a large, distributed mechanical structure. Setting that concern aside for the moment, one can follow the standard quantum mechanical protocol to establish that the energy of each mode is quantized: $E=\hbar \omega(N+1 / 2)$, where $N=0,1,2, \ldots$ is the occupation factor of the mechanical mode of angular frequency $\omega$. The 
a

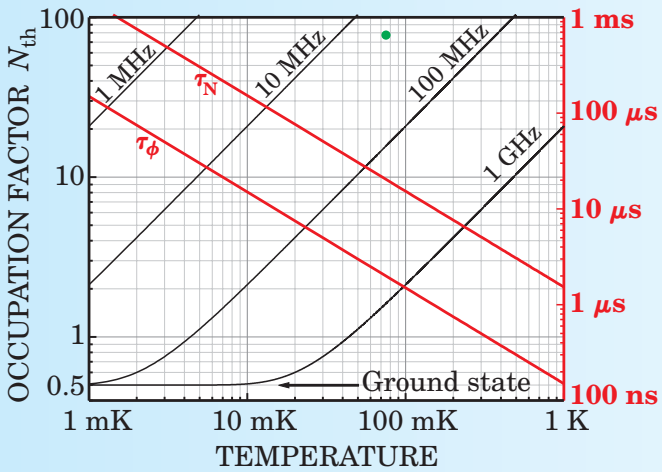

b

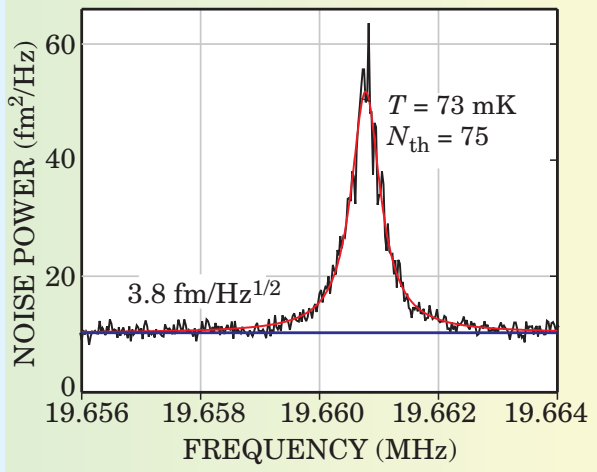

Figure 2. Quantum limits. (a) The occupation factor $N_{\text {th }}$ (black curves) of various mechanical resonator frequencies is a function of resonant frequency and temperature $T$. Shown in red is the lifetime $\tau_{\mathrm{N}}$ of a given number state for a $10-\mathrm{MHz}$ resonator with quality factor $\mathrm{Q}=200000$ (recently demonstrated at the Laboratory for Physical Sciences). ${ }^{11}$ Also in red is the expected decoherence time $\tau_{\phi}$ for a superposition of two coherent states in that resonator displaced by $100 \mathrm{fm}$. (b) The measured noise-power spectrum of the thermal motion (black line, with a Lorentzian fit in red) atop the white noise (blue baseline) of the position detector. The curve corresponds to the green point in panel a, with $T=73 \mathrm{mK}$ and $N_{\text {th }}=75$. These data show the closest approach to date to the uncertainty-principle limit: The detector noise gives a displacement sensitivity a factor of 5.8 from the quantum mechanical limit.

quantum ground state, $N=0$, has a zero-point energy of $\hbar \omega / 2$ and is described by a Gaussian wavefunction of width $\left\langle x^{2}\right\rangle^{1 / 2}=\Delta x_{\mathrm{SQL}}=\sqrt{\hbar /(2 m \omega)}$. This quantity, known as the standard quantum limit, is the root mean square amplitude of quantum fluctuations of the resonator position. ${ }^{7}$ The larger the zero-point fluctuations, the easier they are to detect. For example, a radio-frequency $(10-30 \mathrm{MHz})$ nanomechanical resonator, with a typical mass around $10^{-15} \mathrm{~kg}$, has $\Delta x_{\mathrm{SQL}}=10^{-14} \mathrm{~m}$, some $10^{5}-10^{6}$ times larger than $\Delta x_{\mathrm{SQL}}$ for the macroscopic test masses in the gravitationalwave detectors. Although $10^{-14} \mathrm{~m}$ represents a distance only a little larger than the size of an atomic nucleus, it is readily detectable by today's advanced methods, such as those described below. At the other extreme, a carbon nanotube $1 \mu \mathrm{m}$ long has $\Delta x_{\mathrm{SQL}} \approx 10^{-10} \mathrm{~m}$, about the size of a small atom. That relatively large value, although small by absolute standards, makes nanotubes and nanowires very attractive for displaying and exploring quantum phenomena with mechanical systems.

A crucial consideration for reaching the quantum limit of a mechanical mode is the thermal occupation factor $N_{\text {th }}$, set by the mode frequency $\omega$ and the device temperature $T$. The average fluctuating energy of an individual mechanical mode coupled to a thermal bath is expected to be

$$
\langle E\rangle=\hbar \omega N_{\text {th }}=\hbar \omega\left(\frac{1}{2}+\frac{1}{e^{\hbar \omega / k_{\mathrm{B}} T}-1}\right),
$$

where $N_{\text {th }}$ follows the Bose-Einstein distribution. For high temperatures this expression reduces to the classical equipartition of energy: Each mode carries $k_{\mathrm{B}} T$ of energy. Figure 2a displays the deviation from classical behavior that occurs at low temperatures: When $k_{\mathrm{B}} T \ll \hbar \omega, N_{\text {th }}$ is less than 1 and the mode becomes "frozen out." For a 1-GHz resonator, this freeze-out occurs for $T<50 \mathrm{mK}-\mathrm{a}$ regime well within the range of standard dilution refrigerators employed in low-temperature laboratories. Furthermore, a 1-GHz device can be readily shielded from parasitic external driving forces. Nanomechanical resonators with a fundamental flexural resonance exceeding $1 \mathrm{GHz}$ have been demonstrated by one of us (Roukes) at Caltech, ${ }^{1}$ al- though position-detection schemes with the bandwidth and sensitivity to measure the tiny amplitude of the zero-point fluctuations are still under development.

Fluctuations, both thermal and quantum, are of great practical and fundamental importance: They set ultimate limits for sensitive force detection and for the coherence time of quantum states. These limits are determined by the nanoresonator quality factor $Q$, which parameterizes the coupling strength to the thermal bath. Currently, all atomic-force microscopes and experiments are in the high-temperature limit: $k_{\mathrm{B}} T \gg \hbar \omega$ and $\langle E\rangle \approx k_{\mathrm{B}} T$ per mode. For an acousticfrequency mechanical resonator typically found in an atomic-force microscope setup, with a resonant frequency around $5 \mathrm{kHz}$, a mass of $10^{-12} \mathrm{~kg}$, and a quality factor $Q$ on the order of $10^{4}$, one finds at room temperature that $N_{\text {th }}=10^{9}$ and the $\mathrm{rms}$ displacement fluctuations, $\sqrt{\left\langle x_{\mathrm{th}}^{2}\right\rangle}=10^{-9} \mathrm{~m}$, are more than $10^{4}$ times larger than $\Delta x_{\mathrm{SQL}}$ for this device. Thermal fluctuations limit the force sensitivity to $240 \mathrm{aN} / \mathrm{Hz}^{1 / 2}$ (where $1 \mathrm{aN}=10^{-18} \mathrm{~N}$ ). By cooling a very thin and compliant low-frequency cantilever to near $200 \mathrm{mK}$, the IBM group has been able to reduce the fluctuations to $6 \times 10^{-13} \mathrm{~m}$ and achieve a record force sensitivity of $0.8 \mathrm{aN} / \mathrm{Hz}^{1 / 2}$.

Despite the close analogy between the quantization of the motion of an extended mechanical device and the quantization of the electromagnetic field in a cavity, some important fundamental differences exist. The total zero-point energy in a mechanical system is finite because the number of mechanical degrees of freedom is finite. Also, even for a rectangular flexural resonator, the mechanical mode structure is very complex and differs from the harmonic structure for a rectangular electromagnetic cavity resonator. In addition, due to the resonator's finite stretching and consequent tensioning, there is an intrinsic mechanical nonlinearity that is easily observable for modest mechanical amplitudes. The nonlinearity provides a mode-mode coupling, analogous to photon-photon coupling in nonlinear dielectrics, and drives instabilities. This nonlinearity has already been put to effective use for both parametric mechanical amplification and square-law mechanical detection, as described below.

\section{The challenge of motion transduction}

A prerequisite for attaining the ultimate potential from nanomechanical devices is displacement sensing-that is, reading out the NEMS motional response induced by an applied stimulus. Most typically, this requirement distills to transduction, or conversion, between the mechanical and electrical domains: Ultimately one wants a voltage signal that provides the time record of the NEMS response. Many displacement-sensing techniques for mechanical devices have been proposed and demonstrated (see figure 3); perhaps not surprisingly, displacement transducers that 
Figure 3. Detection techniques for nanomechanical displacement. (a) An optical detection scheme that is typically used in atomic-force microscopy but that does not work well for NEMS. Light from an optical fiber reflects off the resonator and returns up the fiber. (b) A magnetomotive technique that measures the electromotive force generated when the metal layer on top of the resonator moves in the magnetic field B. (c) Coupling to a mesoscopic detector such as a quantum dot, a quantum point contact, or a singleelectron transistor. The current $I_{\mathrm{DS}}$ through the detector is modulated by the NEMS motion.

are important for microscale devices do not prove optimal for nanoscale devices. For example, such optical methods as fiber-optic interferometry and reflecting off a cantilever (the so-called optical lever) have been used extensively in MEMS, especially on commercially available scanning probe microscopes. However, because nanomechanical devices are small compared to the wavelength of light, diffraction dramatically complicates such approaches. Nearfield optical methods may play an important future role. But optical adsorption and the resulting heating of the mechanical structure prove problematic for the most sensitive regimes. So it appears that optimal coupling at the quantum limit may be difficult to achieve optically. Nevertheless, optical techniques have recently enabled the detection of microcantilever motion induced by a single electron spin. ${ }^{3}$

Piezoresistive displacement transduction has found widespread applications in larger micron-scale devices. Use of this technique for nearly quantum-limited detection of NEMS devices, however, does not appear to be straightforward. The dissipation involved will cause appreciable heating of tiny devices, which will likely preclude operation at ultralow temperatures.

One technique that has been immensely successful is magnetomotive detection, which utilizes the electromotive force generated by a metallic conductor (typically a metallization layer) affixed to a mechanical device that moves in a large magnetic field-on the order of several tesla. This technique is especially well suited for low-temperature measurements for which the imposition of large magnetic fields is commonplace; it has been employed to monitor the highest-frequency devices measured to date, with fundamental flexural modes exceeding $1 \mathrm{GHz} .{ }^{1}$ Unfortunately, magnetomotive motion detection becomes increasingly awkward at higher frequencies. The effective electrical impedance, which arises from electromechanical coupling to the motional part of the device, scales inversely with frequency and hence tends to be swamped by parasitic, static circuit impedances as the frequency increases into the gigahertz domain. But even at lower frequencies, at hundreds of megahertz, observing the thermal fluctuations of a nanomechanical resonator by this technique has so far proven elusive - the intrinsic noise of state-of-the-art cryogenically cooled amplifiers is too high to surmount the bugaboo of insufficient mechanical-to-electrical transduction efficiency. The problem is generic for most motiontransduction methods at microwave frequencies.

Using parametric amplification, the Caltech group has demonstrated an attractive way to work around this problem. They employ the Euler instability-the nonlinearity that causes a beam under compression to buckleto realize a high-frequency amplifier that works entirely on mechanical principles. Their device, depicted in figure $1 d$, provides stable gain and up to a thousandfold displacement amplification in response to a weak stimulus force at $17 \mathrm{MHz}$. With sufficient gain in the mechanical do-
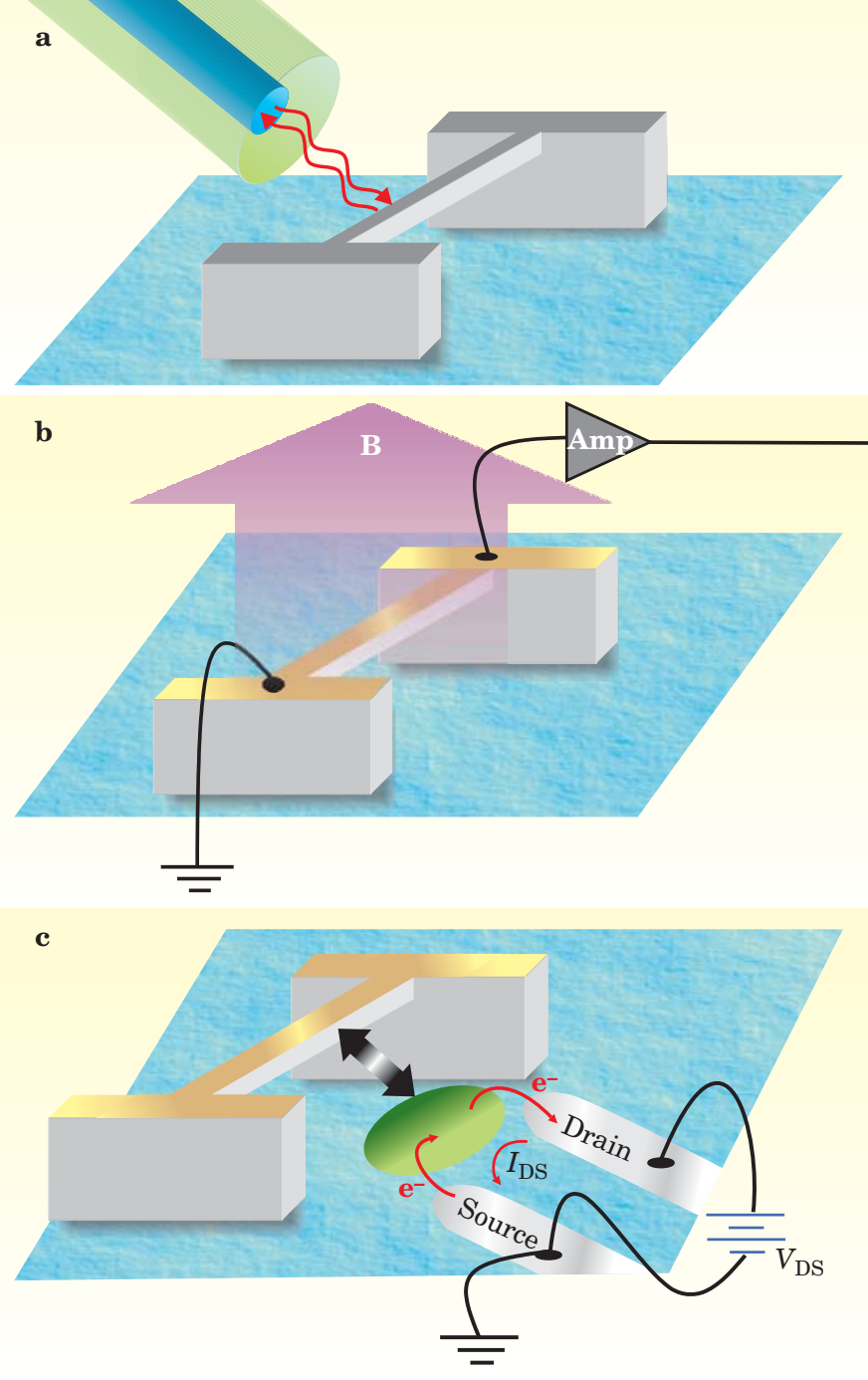

main, the challenge of displacement transduction becomes substantially easier and thermomechanical fluctuations in a cryogenically cooled device have indeed been observed with such an amplifier.

Some of the most exciting recent transduction efforts are focused on coupling high-frequency nanomechanical systems to various nanoelectronic and mesoscopic devices that serve essentially as integrated amplifiers. Examples are quantum point contacts, quantum dots, or single-electron transistors (SETs) used in configurations where the motion of a nanomechanical device modulates the electron transport properties. Among these readout strategies, the SET - shown by many research groups to be a very sensitive detector of charge-has now enabled nearly quantumlimited position detection for NEMS devices. Figures 1a and 1e each show a charged nanomechanical resonator capacitively coupled to an SET. The resonator's motion induces a change in the charge on the gate electrode of the SET; the resulting change in the SET's conductance can be directly monitored. Careful consideration of noise sources indicates that the SET can provide motion detection with sensitivity down to the quantum limit.

Robert Knobel and Andrew Cleland at the University of California, Santa Barbara, ${ }^{10}$ were the first to demonstrate position detection using an SET. They used the device as a narrowband mixer-with a bandwidth of only $100 \mathrm{~Hz}$ - and detected the flexural resonance of a $100-\mathrm{MHz}$ device. Their effort yielded continuous position detection 
to within a factor of about 100 of the quantum limit, but their detection bandwidth was very limited.

Recently a group led by one of us (Schwab) at the Laboratory for Physical Sciences has shown that a radiofrequency SET can simultaneously provide both large bandwidth $(75 \mathrm{MHz})$ and ultrasensitive detection. ${ }^{11}$ The group directly detected the motion of a $20-\mathrm{MHz}$ resonator (figure 1a), which had a $Q$ of 50000 , and demonstrated nanomechanical measurements only a factor of about 6 from the quantum limit, the closest approach to date for any position measurement (see figure $2 \mathrm{~b}$ ). With such position sensitivity, they observed random vibrations driven by the thermal energy at an effective temperature as low as $60 \mathrm{mK}$. The experiment essentially constituted millikelvin noise thermometry on a single mechanical degree of freedom. At the experiment's lowest temperature, the thermal occupation factor $N_{\text {th }}$ for the mechanical mode was on the order of 58; that low value indicates that the quantum ground state $\left(N_{\text {th }}<1\right)$ of NEMS devices is within reach. Necessary steps toward achieving that goal include optimized transduction at higher resonant frequencies and improvements in thermalization, that is, the cooling of the mechanical mode.

Cooling is less straightforward than one might initially assume. Precisely in the regime where device modes become frozen out (and quantum effects begin to emerge), thermal conductance quantization (see PHYSICS TODAY, June 2000, page 17) imposes limits on the rate at which a nanoscale device can thermalize to its environment. ${ }^{12}$ Work-arounds are few. One can perhaps attempt to engineer the modes mediating thermal contact-to optimize heat transfer while preserving the quantum nature of the specific mechanical mode under study-but it is not clear how practical this approach will be. Perhaps a more promising alternative is active cooling of the mechanical mode through controllable external interactions. ${ }^{13}$ For example, using very low-noise temperature detection to provide optimal feedback, it should be possible to actively cool the mechanical mode close to the ground state. The prospects are reminiscent of the strategy taken with trapped atoms: Laser-cooling of samples into a low-energy state allows for various quantum measurements before the atoms begin to warm up from their interaction with the environment.

Ultimately, molecular mechanical systems, assembled with atomic precision, will subsume today's nanomechanical systems patterned by top-down methods. Devices based on single-wall carbon nanotubes offer very exciting possibilities because nanotubes are electronically active and naturally form nanoelectronic devices such as singleelectron transistors. Exploiting the strain dependence of electron transport through a carbon nanotube, Paul McEuen's group at Cornell University has recently detected the vibrational mode spectrum of a suspended nanotube (see figure 1f).$^{14}$ Although the "bottom up" fabrication is today still an art rather than a technology, nanotube and nanowire NEMS offer great promise for achieving both very high resonant frequencies and sensitive force detection.

\section{Uncertainty-principle limits on position detection}

How precisely can one continuously measure the position of an object? This basic question is of central importance for mechanical force detection, such as in atomic-force microscopy. It is clear that quantum mechanics should place limits on the ultimate answer, since it's possible to construct from continuous measurement records both the mechanical resonator's position and its momentum. One should not expect to be able to violate the uncertainty principle.

To continuously record the position, one must couple the mechanical device to some form of linear detector; practically, the detector would take the form of a displacement transducer (such as one that linearly converts position to voltage) and an amplifying device. During the development of the maser in the $1960 \mathrm{~s}$, it became clear that the performance of any linear amplifier is limited by the uncertainty principle. In the context of position detection, the ultimate sensitivity was clarified by Carleton Caves and colleagues ${ }^{6}$ : Even for optimal engineering, the measurement record is obscured by an equal contribution of quantum noise from the mechanical resonator plus its transducer and from the subsequent linear amplifier used as a readout. With the resonator at zero temperature, the minimum possible variance of the position record is

$$
\Delta x_{\mathrm{QL}}=\sqrt{\frac{\hbar}{m \omega \ln 3}} \approx 1.35 \Delta x_{\mathrm{SQL}} .
$$

Two distinct forms of noise arise from the readout process and affect nanomechanical measurements. The first, measurement noise, is independent of the resonator and is added to the output signal. In practice, this contribution may take the form of electrical or photon shot noise. The second is the somewhat more subtle back-action noise, which emanates from the readout and drives the resonator stochastically. Back-action noise might arise from fluctuating potentials or currents that are, in turn, converted by the transducer into physical forces subsequently imposed on the mechanical device. Electrical engineers are familiar with these two forms of noise from linear amplifiers and represent them as voltage and current noise, respectively. Optimally engineered coupling between the resonator and the amplifier is achieved when the two amplifier noise contributions are equal. But very few linear amplifiers, even when optimized in this way, can approach the quantum limit. Accordingly, reaching the quantum limit for position detection involves choosing an amplifier that is capable of quantumlimited detection in principle, and that can be optimally coupled to the mechanical resonator in practice.

\section{Coupling nanomechanics to quantum systems}

A large effort in condensed matter physics today is focused on investigating the coherent quantum mechanical behavior of individual solid-state devices. That research began with mesoscopic physics in the 1980s and continues today, motivated by interest in quantum information and computation. Beautiful and convincing quantum behavior-quantized energy, coherent evolution, superposition states, and entanglement-has been observed with various single-electron devices, SQUIDs, and quantum dots. A growing number of researchers now believe that we have the necessary tools to observe similar behavior in small mechanical structures.

To explore quantum effects in NEMS, it is essential to move beyond continuous measurement and linear coupling: Continuous linear measurements cannot distinguish between classical and quantum oscillators. The quantum properties of the most heavily studied simple harmonic oscillator-the modes of the electromagnetic field-have been revealed by coupling to a nonlinear detector such as a photon counter (for energy detection) or to a coherent two-level system, as in a single atom in an electromagnetic cavity.

Analogous measurement concepts in nanomechanics are starting to take shape. Reviewing the experimental status of quantum nondemolition measurements in 1996, Braginsky and Khalili wrote that "no experimental scheme had been proposed so far" for measuring the energy of a mechanical resonator. ${ }^{7}$ The Caltech group has set 


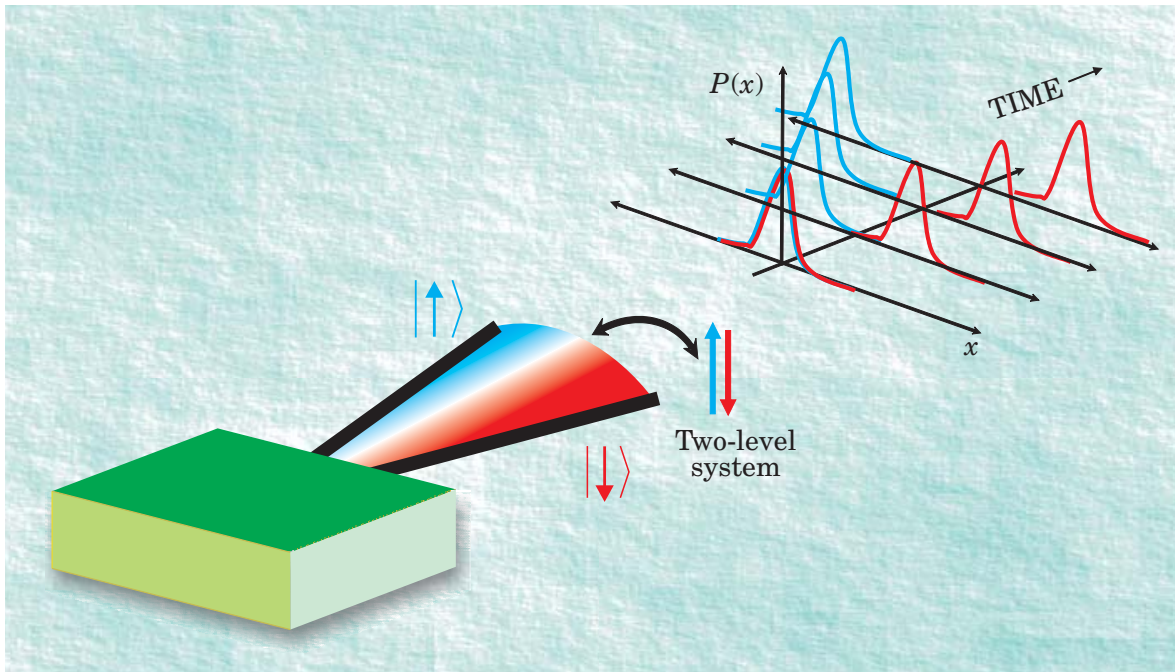

Figure 4. Quantum superpositions may be possible with a quantum two-level system (TLS) coupled to a nanomechanical resonator. In this schematic, the flexure of the cantilever depends on whether the TLS is in the down state $|\downarrow\rangle$ (red) or the up state $|\uparrow\rangle$ (blue). The plot shows the probability $P$ of finding the resonator center-ofmass at a given position $x$ for different times after coupling the resonator to the TLS. If the TLS is prepared in a superposition state, an entangled state is formed and the resonator will be in both locations.
The CPB and the mechanical resonator interact simply through the electrostatic force mediated by the charge on the box. The coupling strength can be comparable to the resonator quantization energy for reasonable parameters. Adding in the single-mode harmonic-oscillator Hamiltonian that describes the mechanical resonator, the system is directly analogous to cavity quantum electrodynamics. In fact, for certain CPB biases, the system is described by a Hamiltonian (the so-called JaynesCumming Hamiltonian) widely employed throughout atomic physics. Various groups exploring this analogy have concluded that the NEMS-CPB system should allow for experiments such as number-state detection, quantum nondemolition measurements, and a resonator cooling process analogous to laser cooling of atoms.

These simple descriptions of

about to change that; recently they demonstrated how to build a mechanical square-law detector-whose output is proportional to the square of the position coordinateanalogous to Braginsky and Khalili's energy detector. Experimental work on this front is under way, with theoretical investigation led by researchers at Caltech and the University of Queensland. ${ }^{15}$

The detection of a single electron spin by Rugar's IBM group is the first realization of a single quantum two-level system coupled to a mechanical mode. ${ }^{3}$ This success demonstrates both that the coupling between the cantilever and spin can be strong enough to produce measurable displacements and that the coherence of the spin is sufficient to perform many coherent manipulations, even while the spin is coupled to the resonator. A positive-feedback scheme appears possible: The coherent back action of the cantilever onto the spins can directly stimulate the transition of more spins and give rise to amplified cantilever oscillations, a mechanical lasing scheme dubbed the cantilaser. ${ }^{16}$ This proposed technique has the advantage that the dynamics of the cantilever-spin system is not limited to the mechanical relaxation time $\tau_{m}=Q / \omega_{0}$, and it may prove useful in the fast mechanical detection of spin ensembles.

The quantum system formed by capacitively coupling a NEMS resonator to a solid-state qubit has garnered perhaps the most attention from theorists. Andrew Armour, Miles Blencowe, and one of us (Schwab) first described one such system, a NEMS coupled to a Cooper-pair box. ${ }^{17}$ The CPB is a superconducting device in which both the electrostatic charging energy of a small conducting island and the Josephson coupling energy are important in the coherent quantum dynamics of the device's charge states. For certain bias configurations, the CPB is well described by a simple two-level qubit Hamiltonian in which the island's capacitance determines the spacing between the levels $|0\rangle$ and $|1\rangle$, which have zero and one extra Cooper pair, respectively; the Josephson energy determines the transition rate between the two charge states. Experiments by a number of researchers have demonstrated CPB eigenstates with excitedstate lifetimes of up to $7 \mu \mathrm{s}$ and coherence times of superposition states as long as $0.5 \mu \mathrm{s}$. coupled quantum systems suggest an intriguing possibility, first described by Armour and colleagues. ${ }^{17}$ The interaction displaces the resonator according to the charge state of the CPB: The device moves to the left if the CPB is in the $|0\rangle$ charge state, and moves to the right if the CPB is in the $|1\rangle$ charge state. This coupling raises the simple question, Which way does the mechanics move if one prepares the CPB into the superposition state $(|0\rangle+|1\rangle) / \sqrt{2}$ ? If the mechanics truly is quantum, then naively one expects the resonator to displace in both directions and ultimately form an entangled state with the qubit (see figure 4).

To design and fabricate experimental devices that allow for such observations, at least two criteria need to be satisfied. First, the coupling between the qubit and the resonator must be sufficient so that the difference in displacement generated by the two qubit states should be greater than $\Delta x_{\mathrm{SQL}}$. Meeting this condition will ensure that an entangled state is formed with two nearly orthogonal positions of the resonator. Second, the decoherence time of the resonator must be larger than the measurement time. The decoherence time of a state formed by a superposition of two coherent states displaced by a distance $\Delta x$ and coupled to a thermal bath modeled by simple harmonic oscillators was calculated by Wojciech Zurek and colleagues at Los Alamos National Laboratory in 1992. Their result,

$$
\tau_{\phi}=Q \frac{\hbar}{k_{\mathrm{B}} T}\left(\frac{\Delta x_{\mathrm{SQL}}}{\Delta x}\right)^{2},
$$

is plotted in figure $2 \mathrm{a}$. For a very small displacement of the two superposition states, $\Delta x=3 \Delta x_{\mathrm{SQL}}=100 \mathrm{fm}$, for which the states are still quantum mechanically distinct, $\tau_{\phi}$ is expected to be surprisingly long-greater than $1 \mu \mathrm{s}$ at $100 \mathrm{mK}$. For an entangled state with $\Delta x$ comparable to the width of a typical resonator, $100 \mathrm{~nm}, \tau_{\phi}$ is estimated to be $10^{-18} \mathrm{~s}$ ! Thus Schrödinger-cat states formed by a mechanical resonator coupled to a qubit appear to be feasible, although the distance between these states would be subatomic.

\section{Illuminating the quantum world}

Nanomechanical resonators will be increasingly useful for explorations of quantum mechanics, whether as ultrasensitive 
probes of quantum and mesoscopic forces, as detectors of single quantum systems, as "buses" in quantum information devices, ${ }^{18}$ or as devices to allow the study of quantum behavior in ordinary bits of matter. The construction of a scanning microscope that can detect single nuclear spins remains a grand challenge of nanomechanics. Success in these areas and others will require highly engineered mechanical structures that routinely operate near quantum mechanical limits.

The generation and detection of the uniquely quantum states of a small mechanical device, such as energy eigenstates (so-called Fock states), superposition states, or entangled states, are particularly interesting because the mechanical structures may be considered "bare systems": There is no macroscopic quantum condensate to protect the device from excitation or decoherence. In superconductors, superfluids, or Bose-Einstein condensates, the number of quantum states of a macroscopic sample is drastically reduced to only a few degrees of freedom. For instance, in a CPB containing billions of electrons, superconductivity suppresses almost all the normal-state degrees of freedom down to just two: number and phase.

The mechanical devices shown in figure 1 are composed of ordinary matter, full of defects and imperfections, and help answer the question: What does it take to observe the quantum nature of an ordinary system? The motivation is similar to that behind the recent beautiful work of Anton Zeilinger and colleagues at the University of Vienna, who have shown matter-wave interference with molecules of increasing complexity and have studied decoherence by directly controlled interactions. In this vein, we hope experiments with nanomechanical devices at the quantum limit may further illuminate the boundary be- tween the microscopic realm, governed by quantum mechanics, and our macroscopic world, governed by classical mechanics.

\section{References}

1. X. M. H. Huang et al., Nature 421, 496 (2003).

2. C. Bustamante et al., Annu. Rev. Biochem. 73, 705 (2004); C. Friedsam et al., J. Phys. Condens. Matter 15, S1709 (2003); M. Benoit, H. E. Gaub, Cells Tissues Organs 172, 174 (2002).

3. D. Rugar et al., Nature 430, 329 (2004).

4. K. L. Ekinci, Y. T. Yang, M. L. Roukes, J. Appl. Phys. 95, 2682 (2004).

5. For a review, see M. Bordag, U. Mohideen, V. M. Mostepanenko, Phys. Rep. 353, 1 (2001).

6. C. M. Caves, Phys. Rev. D 26, 1817 (1982); C. M. Caves et al., Rev. Mod. Phys. 52, 341 (1982). A recent treatment from a condensed matter approach has been given by A. A. Clerk, Phys. Rev. B 70, 245306 (2004).

7. V. B. Braginsky, F. Ya. Khalili, Quantum Measurement, Cambridge U. Press, New York (1992).

8. J. A. Sidles et al., Rev. Mod. Phys. 67, 249 (1995).

9. For an excellent review, see M. Blencowe, Phys. Rep. 395, 159 (2004).

10. R. Knobel, A. N. Cleland, Nature 424, 291 (2003).

11. M. D. LaHaye et al., Science 304, 74 (2004).

12. K. C. Schwab et al., Nature 404, 974 (2000).

13. I. Wilson-Rae, P. Zoller, A. Immamoglu, Phys. Rev. Lett. 92, 075507 (2004); A. Hopkins et al., Phys. Rev. B 68, 235328 (2003).

14. V. Sazonova et al., Nature 431, 284 (2004).

15. D. Santamore et al., Phys. Rev. A 70, 052105 (2004).

16. I. Bargatin, M. L. Roukes, Phys. Rev. Lett. 91, 138302 (2003).

17. A. D. Armour, M. P. Blencowe, K. C. Schwab, Phys. Rev. Lett. 88, 148301 (2002).

18. E. K. Irish, K. C. Schwab, Phys. Rev. B 68, 155311 (2003); X. Zou, W. Mathis, Phys. Lett. A 324, 484 (2004); A. N. Cleland, M. R. Geller, Phys. Rev. Lett. 93, 070501 (2004).

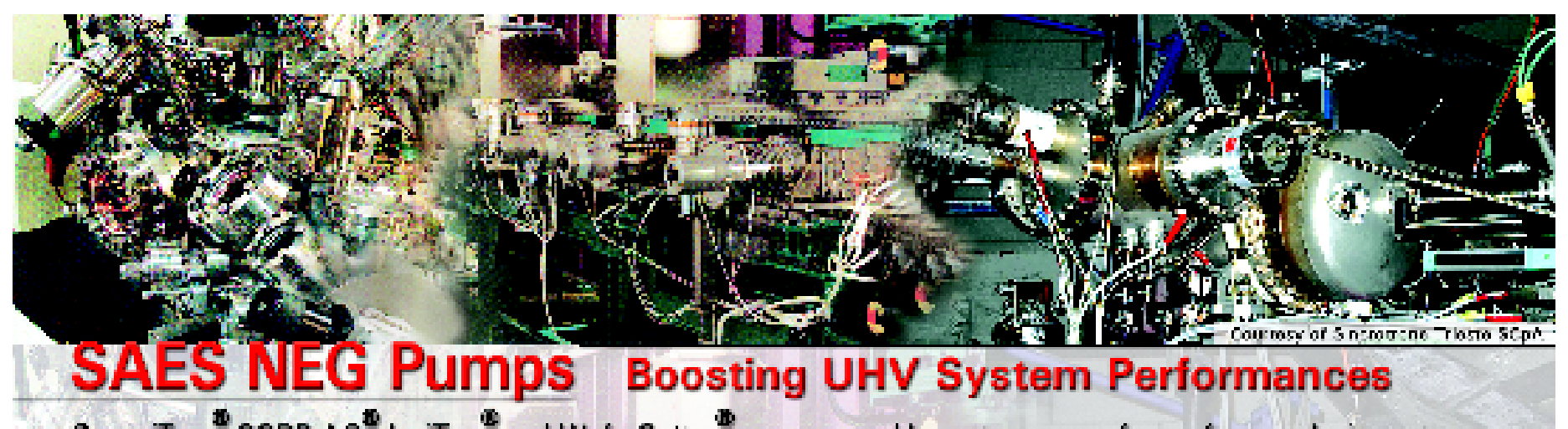

CapaciTorr, SORB-AC." InsiTorr and WaferGetter "non-vaporable getter pumps for surface analysis systems, analytical instrumentation, particle accelerators, multi-purpose UHV systems and thin film deposition equipment
a Faster pumpdowns
a Better base pressure
- Best hydrogen pumping
口 Purmpirng without power
- Cleaner vacuum conditions

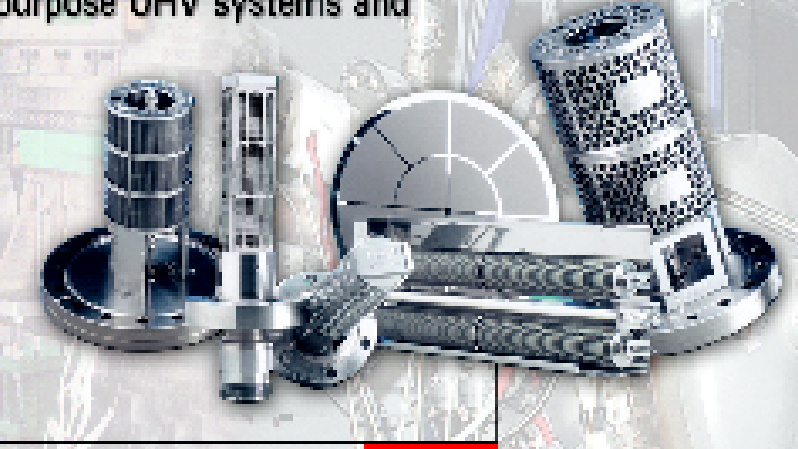

